\title{
Non-local spatial frequency response of photopolymer materials containing chain transfer agents: II. Experimental results
}

\author{
Jinxin Guo, Michael R Gleeson, Shui Liu and John T Sheridan \\ School of Electrical, Electronic and Mechanical Engineering, Communications and \\ Optoelectronic Research Centre, The SFI Strategic Research Cluster in Solar Energy \\ Conversion, College of Engineering, Mathematical and Physical Sciences, University College \\ Dublin, Belfield, Dublin 4, Ireland \\ E-mail: john.sheridan@ucd.ie and jinxin.guo@ucd.ie
}

Received 7 March 2011, accepted for publication 4 August 2011

Published 25 August 2011

Online at stacks.iop.org/JOpt/13/095602

\begin{abstract}
In part I of this paper the non-local photo-polymerization driven diffusion model was extended to include the kinetics of chain transfer and re-initiation, in order to analyse the effects of chain transfer agents on the system kinetics and to study their use in reducing the average polymer chain length in free-radical based photopolymer materials. Based on these results, it is proposed that one possible way to improve the material response at high spatial frequency is the addition of chain transfer agents. In this paper, the validity of the proposed model is examined by applying it to fit experimental data for an acrylamide/polyvinyl alcohol (AA/PVA) layer containing two different types of chain transfer agent (CTA): sodium formate (HCOONa) and 1-mercapto-2-propanol $\left(\mathrm{CH}_{3} \mathrm{CH}(\mathrm{OH}) \mathrm{CH}_{2} \mathrm{SH}\right)$. The effects on decreasing the average polymer chain length formed, by the addition of chain transfer agent, which in turn reduces the non-local response of the material, are demonstrated. These reductions are shown to be accompanied by improved high spatial frequency response. Key material parameters are extracted by numerically fitting experimentally measured refractive index modulation growth curves using the model. Further independent experimental confirmation of the reduction in the average polymer molecular weight is provided using a diffusion based holographic technique.
\end{abstract}

Keywords: holographic data storage, photopolymer material, chain transfer agent, spatial frequency response

(Some figures in this article are in colour only in the electronic version)

\section{Introduction}

In part I [1], a general model was presented, which includes the effects of (i) the kinetics of the major photochemical processes, (ii) the temporally and spatially varying photon absorption, (iii) the kinetics of chain transfer, and (iv) the non-local material response. Applying appropriate initial conditions and material parameter values, simulations were carried out using the extended non-local photo-polymerization driven diffusion (NPDD) model. It was shown that by changing the CTA present (concentration and kinetic properties), the average polymer chain length could be controlled. The effects of the non-local response length on a photopolymer material's performance were examined. It was shown that reducing the non-locality of the polymer chains' growth will improve the spatial frequency response of the material [2]. Based on these two results it was concluded in part I that a controlled decrease in average polymer chain length would result in a lower non-local length, $\sqrt{\sigma}$, and therefore an improved high spatial frequency response. One chemical way to achieve such a reduction in non-local chain growth is to introduce chain transfer agents (CTAs) into a free-radical photopolymer system, causing the production of more, shorter polymer chains [2]. 
Table 1. Components and volume fractions of the material produced.

\begin{tabular}{llllllll}
\hline Component & Mass $(\mathrm{g})$ & $\begin{array}{l}\text { Density } \\
\left(\mathrm{g} \mathrm{cm}^{-3}\right)\end{array}$ & $\begin{array}{l}\text { Volume } \\
\left(\mathrm{cm}^{3}\right)\end{array}$ & $\begin{array}{l}\text { Standard } \\
\text { volume fraction }\end{array}$ & \multicolumn{2}{l}{ Volume fraction with CTA } \\
\hline PVA & 7.0000 & 1.300 & 5.3846 & 0.3330 & 0.3280 & 0.3256 & 0.3231 \\
AA & 2.4000 & 1.1220 & 2.1390 & 0.1323 & 0.1303 & 0.1293 & 0.1283 \\
BA & 0.8000 & 1.2400 & 0.6451 & 0.0399 & 0.0393 & 0.0390 & 0.0387 \\
TEA & 8.9920 & 1.1240 & 8.0000 & 0.4947 & 0.4872 & 0.4836 & 0.4799 \\
CTA & - & - & - & - & 0.0152 & 0.0225 & 0.0300 \\
\hline
\end{tabular}

In this paper, the validity and generality of the extended NPDD model are examined by applying it to fitted experimental data following the inclusion of different concentrations of two types of chain transfer agent, sodium formate (CTA-1) and 1-mercapto-2-propanol (95\%) (CTA-2), in an AA/PVA based photopolymer material [2-6]. The model includes the effects of CTAs, which we now verify experimentally, and the best CTA and most appropriate concentration are determined. We note that introduction of a CTA into the photopolymer material causes the premature termination of growing macro-radical chains. Its presence therefore leads to a reduction in the average polymer chain length (molecular weight) formed and an increase in the number of individual polymer chains. In order to determine a suitable concentration of CTA, a diffusion based experimental technique is carried out to examine the effects of including various concentrations of each of the transfer agents tested on the average polymer chain length. Experimental growth curves of unslanted transmission gratings are extracted from the measured diffraction efficiency data, with the most suitable transfer agent concentration being identified by examining the resulting material spatial frequency response. The saturation refractive index modulations for each type of material layer and for a range of spatial frequencies are recorded and discussed. Furthermore, in order to examine the effects of the addition of chain transfer agents at very high spatial frequencies, holographic gratings are recorded in reflection geometry.

This paper is organized as follows. In section 2, the volume fraction of each component in the material layer and their respective refractive index values are reviewed, and are described using the Lorentz-Lorenz relation. A polymer diffusion model is developed and used to examine the effects on the polymer chain length of varying the concentrations of each chain transfer agent in order to determine the optimum material composition. In section 3 , the diffraction efficiencies of each of the gratings recorded in the various material compositions are examined for a large range of spatial frequencies. The refractive index modulations and thicknesses of the material layers are then evaluated by fitting the experimental curves obtained from angular scans of the gratings. Then, using the model presented in part I [1], key material parameters are extracted by numerically fitting the experimental refractive index modulation growth curves obtained. Following this, the non-local response length is determined for a range of spatial frequencies and the optimum CTA identified. A brief conclusion is presented in section 4 .

\section{Material components and polymer diffusion}

Our standard dry photopolymer layer typically consists of monomer (acrylamide-AA), binder (polyvinyl alcoholPVA), cross-linker (bisacrylamide-BA), an electron donor (triethanolamine-TEA) and a photo-initiator (erythrosine $\mathrm{B}-\mathrm{EB})$ which is sensitive at $\lambda=532 \mathrm{~nm}$ [7]. Table 1 lists the composition of this standard material with and without the presence of the chain transfer agent examined, including the mass, density, and volume of each component. The photosensitizer is not listed in this table due to the low concentration present relative to the overall material composition [2]. To prepare dry material layers for holographic recording the solution prepared above is used as follows.

(a) The glass substrate on which the material is to be deposited (approximately $5 \times 4 \mathrm{~cm}^{2}$ ) is cleaned thoroughly using de-ionized water and acetone. Once cleaned the plates are placed on a level surface so that the photopolymer layers will adhere to the glass evenly, producing a layer of uniform thickness.

(b) $2 \mathrm{ml}$ of the photopolymer solution is then deposited evenly over the area of the glass plate using a syringe.

(c) Using this method, the typical material thickness is $110 \pm 10 \mu \mathrm{m}$. Different thicknesses can be obtained by depositing (drop casting) different quantities of material. The thickness and uniformity of these layers can be measured using a micrometre screw gauge.

(d) The plates are then left in the dark for approximately 24 h until dry ( $\sim 96 \%$ water content has evaporated). The drying time is dependent on the thickness of the material and the relative humidity.

(e) Once dry the plates are stored in a lightproof dessicator, ready for use.

\subsection{Volume fraction analysis}

Following the analysis presented in [8, 9], and using equation (29) in [1], the refractive indices of the individual material components and their volume fractions, which determine the average refractive index of the material, $n$, can be expressed using the Lorentz-Lorenz relation:

$$
\begin{gathered}
\frac{n^{2}-1}{n^{2}+2}=\phi^{(\mathrm{AA})} \frac{n_{\mathrm{AA}}^{2}-1}{n_{\mathrm{AA}}^{2}+2}+\phi^{(\mathrm{PVA})} \frac{n_{\mathrm{PVA}}^{2}-1}{n_{\mathrm{PVA}}^{2}+2}+\phi^{(\mathrm{BA})} \frac{n_{\mathrm{BA}}^{2}-1}{n_{\mathrm{BA}}^{2}+2} \\
+\phi^{(\mathrm{TEA})} \frac{n_{\mathrm{TEA}}^{2}-1}{n_{\mathrm{TEA}}^{2}+2}+\left(\phi^{(\mathrm{CTA})} \frac{n_{\mathrm{CTA}}^{2}-1}{n_{\mathrm{CTA}}^{2}+2}\right)
\end{gathered}
$$


Table 2. Contents of chain transfer agents and calculated refractive index values of the material components. CTA-1 is sodium formate and CTA-2 is 1-mercapto-2-propanol.

\begin{tabular}{lllll}
\hline $\begin{array}{l}\text { CTA } \\
\text { [refractive index }]\end{array}$ & $\begin{array}{l}\text { Mass } \\
(\mathrm{g})\end{array}$ & $\begin{array}{l}\text { Density } \\
\left(\mathrm{g} \mathrm{cm}^{-3}\right)\end{array}$ & $\begin{array}{l}\text { CTA volume } \\
\text { fraction }\end{array}$ & $n_{\text {dark }}$ \\
\hline Sodium formate & 0.480 & 1.920 & 0.0152 & 1.4911 \\
(CTA-1) & 0.640 & & 0.0225 & 1.4910 \\
[1.4623] & 0.960 & & 0.0300 & 1.4907 \\
1-mercapto-2- & 0.270 & 1.048 & 0.0152 & 1.4915 \\
propanol (CTA-2) & 0.353 & & 0.0225 & 1.4915 \\
[1.4860] & 0.53 & & 0.0300 & 1.4914 \\
\hline
\end{tabular}

where $n_{\mathrm{AA}}, n_{\mathrm{PVA}}, n_{\mathrm{BA}}, n_{\mathrm{TEA}}$ and $n_{\mathrm{CTA}}$ are the refractive indices of the monomer (AA), the binder (PVA), the cross-linker (BA), the electron donor (TEA), and the chain transfer agent (CTA), respectively. $\phi^{(\mathrm{AA})}, \phi^{(\mathrm{PVA})}, \phi^{(\mathrm{BA})}, \phi^{(\mathrm{TEA})}$ and $\phi^{(\mathrm{CTA})}$ are the respective volume fractions of these components. As noted in part I [1], polymerization and diffusion will result in some shrinkage and swelling during and post exposure. However, we assume that the total volume fraction is approximately conserved during exposure [8-11]:

$$
\phi^{(\mathrm{AA})}+\phi^{(\mathrm{PVA})}+\phi^{(\mathrm{BA})}+\phi^{(\mathrm{TEA})}+\phi^{(\mathrm{CTA})}=1 .
$$

Table 1 shows the compositions of the materials tested. Applying equations (1) and (2), the various concentrations and volume fractions of the CTAs were determined along with the refractive indices of photopolymer layers before photopolymerization, i.e., $n_{\text {dark }}$. These values are presented in table 2. Furthermore we note that the CTAs have the smallest volume fraction contributions listed in tables 1 and 2, and will therefore have little direct effect on these bulk material properties, i.e., on the average refractive index [2].

Throughout exposure, the amount of polyacrylamide increases with time. The resulting temporal evolution of the refractive index modulation can be expressed by

$$
\begin{aligned}
n_{1}(t) & =\frac{\left(n_{\mathrm{dark}}^{2}+2\right)^{2}}{6 n_{\mathrm{dark}}}\left[\phi_{1}^{(\mathrm{m})}(t)\left(\frac{n_{\mathrm{m}}^{2}-1}{n_{\mathrm{m}}^{2}+2}-\frac{n_{\mathrm{b}}^{2}-1}{n_{\mathrm{b}}^{2}+2}\right)\right. \\
& \left.+\phi_{1}^{(\mathrm{p})}(t)\left(\frac{n_{\mathrm{p}}^{2}-1}{n_{\mathrm{p}}^{2}+2}-\frac{n_{\mathrm{b}}^{2}-1}{n_{\mathrm{b}}^{2}+2}\right)\right],
\end{aligned}
$$

where $n_{\mathrm{b}}$ is the refractive index of the background, which consists of each component without the monomer or polymer present measured at the replay wavelength. It is once again assumed that the total volume fraction is conserved, i.e., $\phi^{(\mathrm{m})}(t)+\phi^{(\mathrm{p})}(t)+\phi^{(\mathrm{b})}(t)=1$, where $\phi^{(\mathrm{m})}(t), \phi^{(\mathrm{p})}(t)$ and $\phi^{(\mathrm{b})}(t)$, are the volume fractions of monomer, polymer and background respectively, [8-11]. In equation (3), $\phi_{1}^{(\mathrm{m})}(t)$ and $\phi_{1}^{(\mathrm{p})}(t)$ are the time varying first harmonic volume fraction components of monomer and polymer respectively. These time varying volume fractions are calculated using the model [9]. In the analysis presented here, the refractive index values are $n_{\mathrm{m}}=1.4719, n_{\mathrm{b}}=1.4957$ and $n_{\mathrm{p}}=1.5020[2,8]$. All the refractive index modulation values presented in this paper were measured using a refractometer at $\lambda=633 \mathrm{~nm}$, the replay probe wavelength.
It must be noted at this point that the contribution due to the refractive index of the CTA is minimal, i.e., appearing in the fourth decimal place, and therefore does not have a significant impact on the overall refractive index of the material. The analysis carried out in this section, which includes the presence of the CTA, supports the conclusion that these effects are negligible, and their direct contribution to any improvement in the material spatial frequency response will be insignificant.

\subsection{Average polymer chain length}

The numerical results in part I [1] indicate that a reduction of the non-local response length, $\sqrt{\sigma}$, leads to an improvement in the high spatial frequency response of a photopolymer material. As noted, reducing the average polymer chain length should reduce the $\sqrt{\sigma}$. Therefore, if a controlled reduction of the average polymer chain length can be achieved chemically, the spatial frequency response of a photopolymer material can be improved. It has been shown in many polymerization systems that the average polymer chain length (molecular weight) is lower in the presence of CTA [2-6, 12-14]. In order to identify a suitable CTA and to determine the optimum concentration, we now introduce a polymer diffusion model to examine the effects of the transfer agent on the average molecular weight of polymer chain formed during exposure.

2.2.1. Polymer diffusion model. It is well known that the average molecular weight of a given polymer has a major effect on the material's physical and mechanical properties. In order to fully characterize a polymer sample, an indication of the distribution of average molecular weights is necessary. We assume a polydisperse polymer system that is made up of polymer chains of similar molecular weights and having similar properties [14]. We attempt to model the situation where more than one polymer diffusion rate is present in order to characterize such a polydisperse polymer sample. Therefore, we assume that the polymer molecules generated during exposure in an AA/PVA material can be divided into $i$ groups, where $\sum_{i} \varphi_{i}=1$, with $\varphi_{i}$ being the molecular fraction of the $i$ th group. Each group represents polymer chains of different average lengths having different average molecular weights and different average rate constants of diffusion, $D_{N, i}$. Hence, equation (3) can be re-expressed as

$$
\begin{aligned}
n_{1}(t) & =\frac{\left(n_{\text {dark }}^{2}+2\right)^{2}}{6 n_{\text {dark }}}\left[\phi_{1}^{(\mathrm{m})}(t)\left(\frac{n_{\mathrm{m}}^{2}-1}{n_{\mathrm{m}}^{2}+2}-\frac{n_{\mathrm{b}}^{2}-1}{n_{\mathrm{b}}^{2}+2}\right)\right. \\
& \left.+\sum \phi_{1}^{(\mathrm{p}), i}(t)\left(\frac{n_{\mathrm{p}}^{2}-1}{n_{\mathrm{p}}^{2}+2}-\frac{n_{\mathrm{b}}^{2}-1}{n_{\mathrm{b}}^{2}+2}\right)\right],
\end{aligned}
$$

where $\phi_{1}^{(\mathrm{p}), i}(t)$ is the volume fraction contribution to the first polymer harmonic corresponding to the $i$ th polymer group. In this section, we aim to examine and compare the effects of the addition of varying concentrations of chain transfer agents on these physical properties. In order to simplify the model, it is next assumed that the number of groups of average polymer molecular weight can be reduced to two, $i=2$ (small molecular weight and large molecular weight), and that the refractive index of the polymer in each group can be treated 
Table 3. The rates of polymer diffusion extracted from the fitting.

\begin{tabular}{llllll}
\hline Composition & $\begin{array}{l}\text { CTA volume } \\
\text { fraction }\end{array}$ & $\begin{array}{l}\text { Large polymer } \\
\text { fraction, } \varphi_{\mathrm{L}}\end{array}$ & $\begin{array}{l}D_{\mathrm{N}, \mathrm{S}}\left(\times 10^{-13}\right) \\
\left(\mathrm{cm}^{2} \mathrm{~s}^{-1}\right)\end{array}$ & $\begin{array}{l}D_{\mathrm{N}, \mathrm{L}}\left(\times 10^{-15}\right) \\
\left(\mathrm{cm}^{2} \mathrm{~s}^{-1}\right)\end{array}$ & $\begin{array}{l}\text { MSE } \\
\left(\times 10^{-10}\right)\end{array}$ \\
\hline Standard (no cross-linker) & - & 0.92 & 1.5 & 1.0 & 1.33 \\
CTA-1 & (a) 0.0152 & 0.88 & 4.0 & 1.5 & 2.20 \\
& (b) 0.0225 & 0.79 & 5.2 & 6.5 & 2.18 \\
CTA-2 & (c) 0.0300 & 0.62 & 3.5 & 7.5 & 1.90 \\
& (d) 0.0152 & 0.90 & 2.9 & 1.0 & 0.92 \\
& (e) 0.0225 & 0.81 & 2.0 & 1.0 & 0.95 \\
& (f) 0.0300 & 0.44 & 4.6 & 7.0 & 1.92 \\
\hline
\end{tabular}

as being identical. The equations governing the polymer concentration for each of these polymer groups are

$$
\begin{aligned}
& \frac{\mathrm{d} N_{\mathrm{L}}(x, t)}{\mathrm{d} t}=\varphi_{\mathrm{L}} \int_{-\infty}^{\infty} k_{\mathrm{p}} M^{\cdot}\left(x^{\prime}, t\right) u\left(x^{\prime}, t\right) G\left(x, x^{\prime}\right) \mathrm{d} x^{\prime} \\
&-\frac{\mathrm{d}}{\mathrm{d} x}\left[D_{\mathrm{N}, \mathrm{L}}(x, t) \frac{\mathrm{d} N_{\mathrm{L}}(x, t)}{\mathrm{d} x}\right], \\
& \frac{\mathrm{d} N_{\mathrm{S}}(x, t)}{\mathrm{d} t}=\left(1-\varphi_{\mathrm{L}}\right) \int_{-\infty}^{\infty} k_{\mathrm{p}} M^{\cdot}\left(x^{\prime}, t\right) u\left(x^{\prime}, t\right) G\left(x, x^{\prime}\right) \mathrm{d} x^{\prime} \\
&-\frac{\mathrm{d}}{\mathrm{d} x}\left[D_{\mathrm{N}, \mathrm{S}}(x, t) \frac{\mathrm{d} N_{\mathrm{S}}(x, t)}{\mathrm{d} x}\right],
\end{aligned}
$$

giving the refractive index modulation as

$$
\begin{aligned}
n_{1}(t) & =\frac{\left(n_{\text {dark }}^{2}+2\right)^{2}}{6 n_{\text {dark }}}\left[\phi_{1}^{(\mathrm{m})}(t)\left(\frac{n_{\mathrm{m}}^{2}-1}{n_{\mathrm{m}}^{2}+2}-\frac{n_{\mathrm{b}}^{2}-1}{n_{\mathrm{b}}^{2}+2}\right)\right. \\
& +\phi_{1}^{(\mathrm{p}), \mathrm{L}}(t)\left(\frac{n_{\mathrm{p}}^{2}-1}{n_{\mathrm{p}}^{2}+2}-\frac{n_{\mathrm{b}}^{2}-1}{n_{\mathrm{b}}^{2}+2}\right) \\
& \left.+\phi_{1}^{(\mathrm{p}), \mathrm{S}}(t)\left(\frac{n_{\mathrm{p}}^{2}-1}{n_{\mathrm{p}}^{2}+2}-\frac{n_{\mathrm{b}}^{2}-1}{n_{\mathrm{b}}^{2}+2}\right)\right],
\end{aligned}
$$

where $N_{\mathrm{L}}$ and $N_{\mathrm{S}}$ denote the concentrations of the two polymer groups, i.e., large polymer and small polymer groups. $D_{\mathrm{N}, \mathrm{L}}$, $D_{\mathrm{N}, \mathrm{S}}, \phi_{1}^{(\mathrm{p}), \mathrm{L}}(t)$, and $\phi_{1}^{(\mathrm{p}), \mathrm{S}}(t)$ are the rates of diffusion and the corresponding volume fractions respectively. For simplicity, we introduce a single parameter $\varphi_{\mathrm{L}}$ to determine the time varying fractions of large and small polymer chains, i.e., $\varphi_{\mathrm{L}}$ indicates the percentage of the polymer chains which are large. The first-order coupled differential equations in equations (5) and (6) can be derived in the same manner as presented in [1]. Combining equations (5)-(7), the temporal evolution of $n_{1}$ can be obtained. Here the initial conditions for $N_{\mathrm{L}}$ and $N_{\mathrm{S}}$ are assumed to be $N_{\mathrm{L}, j \geqslant 0}(t=0)=N_{\mathrm{S}, j \geqslant 0}(t=0)=0$.

2.2.2. Examination of polymer diffusion. In this subsection we examine the effects of the addition of the chain transfer agents using the model presented above. For each CTA concentration examined several gratings were recorded at $\Lambda=$ $700 \mathrm{~nm}$ using an exposing intensity of $1 \mathrm{~mW} \mathrm{~cm}^{-2}$, at $\lambda=$ $532 \mathrm{~nm}$, employing the holographic set-up described in detail elsewhere [7, 15]. In all cases the diffraction efficiency of the probe beam $(\lambda=633 \mathrm{~nm}$ ) was monitored during and post exposure. In order to provide a meaningful comparison, the exposure time was chosen to be $t=50 \mathrm{~s}$ for each case, and equal dosages, $\xi=\sqrt{I_{0}} t$, were delivered [16]. The diffracted intensity measurement data, having been corrected for Fresnel reflection loses, were then converted into grating refractive index modulation growth curves using Kogelnik's coupled wave theory [17].

We now examine the effects of the addition of the CTAs. The samples we tested were as follows.

(i) Standard AA/PVA material without cross-linker (no bisacrylamide). (See the row labelled standard in table 3.)

(ii) Standard material without cross-linker including three different volume fractions of sodium formate (CTA-1), (a) 0.0150 , (b) 0.0225 , (c) 0.0300 . (See the row labelled CTA-1 in table 3.)

(iii) Standard material without cross-linker and with three volume fractions of 1-mercapto-2-propanol (CTA-2), (d) 0.0150 , (e) 0.0225 , (f) 0.0300 . (See the row labelled CTA-2 in table 3.)

The purpose of the cross-linker (bisacrylamide) is to bind the polyacrylamide chains, which are formed during the recording process. This helps to reduce the mobility of the polymer chains and ensures the stability of the grating formed. Removing the cross-linker will allow the polyacrylamide chains to diffuse out of the bright regions of the recorded fringe pattern, resulting in a decay of the grating refractive index modulation with time. Another point to note is that when the cross-linker is not included in the material composition the volume fractions of the other components change. For this reason, an equivalent small volume of monomer is added to compensate these changes, thus maintaining the same volume fractions of the other components. All values are as indicated in tables 1 and 2.

The diffusion model was applied to examine the polymer diffusion. A least square algorithm was used to fit the model to the refractive index modulation curves. Using this technique, values for the diffusion rates of the large polymer and small polymer molecular weight groups and the large polymer fraction, $\varphi_{\mathrm{L}}$, were extracted and are presented in table 3. The corresponding MSE values for the fits are also provided to indicate the quality of the fits to the experimental data. As can be clearly observed from table 3 , an increase in the volume fraction of chain transfer agent leads to a decrease in the large polymer fraction, $\varphi_{\mathrm{L}}$. In all cases, the rate constants of diffusion $D_{\mathrm{N}, \mathrm{L}}$ are in the range of $10^{-15} \mathrm{~cm}^{2} \mathrm{~s}^{-1}$ and all $D_{\mathrm{N}, \mathrm{S}}$ values are in the range of $10^{-13} \mathrm{~cm}^{2} \mathrm{~s}^{-1}$. These values indicate that the number of small polymer chains becomes greater as the volume fraction of chain transfer agent present increases, and thus importantly that the inclusion of the CTAs increases the number of small polymer chains. For CTA-1, the $\varphi_{\mathrm{L}}$ values decrease from 0.88 to 0.62 with the increase in volume fraction 


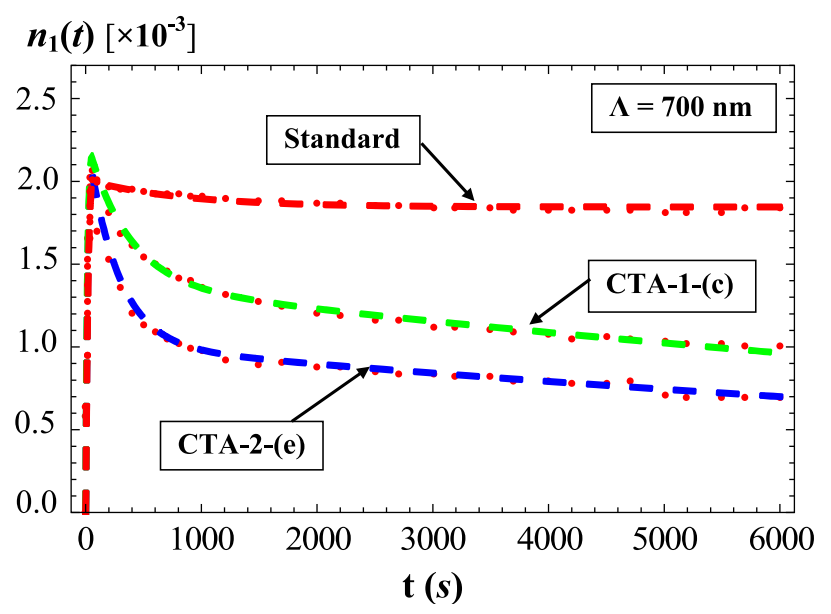

Figure 1. Experimental post-exposure decay curves and fits for standard material (Standard), standard material with the addition of $0.96 \mathrm{~g}$ sodium formate (CTA-1) and standard material with the addition of $0.53 \mathrm{ml} 1$-mercapto-2-propanol (CTA-2).

of sodium formate from 0.0152 to 0.0300 . This corresponds to approximately a $30 \%$ reduction in the fraction of long polymer chains formed, when compared to the standard material composition case. For the case of the addition of CTA-2-(f), the smallest value obtained for $\varphi_{\mathrm{L}}$ was 0.44 , when a volume fraction of 0.0300 was added. Experimentally, it should be noted that there is a sufficiently large reduction in the average molecular weight to allow the effects of the addition of CTA on the average polymer chain length to be clearly visible in figure 1 .

From the results obtained and presented in figure 1 and table 3 , the rate of decay of refractive index modulation obtained for case CTA-2-(f) is faster than the values obtained for all the other samples examined. This indicates that the material composition of case CTA-2-(f) produces polymer chains with the lowest average molecular weight and therefore having the shortest average polymer chain length, and hence should provide the largest reduction in the non-local response parameter. As great care was taken to accurately estimate the $\varphi_{\mathrm{L}}$, values, and since the results obtained are based on averages produced using many reproducible data sets, the effect of the reduction in the non-local response should be most prominent in the material composition corresponding to case CTA-2-(f).

As the analysis indicated in [2], the CTA has little effect on the average refractive index or on the rate of monomer diffusion in the layer. Based on the experimental results and analysis above, it appears as though the most suitable CTA volume fractions to reduce the non-local response length are 0.0300 for each of the CTAs examined. We therefore proceed to examine these material compositions experimentally, producing the spatial frequency response for each.

\section{Spatial frequency response}

In this section, we aim to demonstrate and compare the effects of the CTAs on the spatial frequency responses of the materials under examination. Following the analysis presented in part I, we show that the demonstrated reduction in average polymer chain length reported in section 2 does indeed reduce the non-local chain length variance, $\sqrt{\sigma}$. In order to experimentally confirm this hypothesis, the growth curves and the saturation values of refractive index modulation, $n_{1}$, for each photopolymer material composition, for spatial frequencies from 500 to 4799 lines $\mathrm{mm}^{-1}$, are measured and compared [7, 16].

For each sample material composition tested in this section several growth curves were measured using a recording intensity of $1 \mathrm{~mW} \mathrm{~cm}^{-2}$ at $\lambda=532 \mathrm{~nm}$. All the experiments were repeated several times to ensure their reproducibility. In all cases the diffraction efficiency of a probe beam at $\lambda_{\mathrm{p}}=$ $633 \mathrm{~nm}$ was monitored during and post exposure and angular scans of the gratings were performed and analysed.

\subsection{Diffraction efficiency}

The time varying refractive index modulation, $n_{1}(t)$, can be calculated from the probe beam diffraction efficiency $\eta(t)$ for unslanted transmission geometry grating [17],

$$
n_{1}(t)=\frac{\lambda_{\mathrm{p}} \sin ^{-1} \sqrt{\eta(t)} \cos \theta_{\mathrm{B}}}{\pi d},
$$

where $\lambda_{\mathrm{p}}$ is the replay probe wavelength, $\eta(t)$ is corrected for Fresnel reflection loses, $\theta_{\mathrm{B}}$ is the on-Bragg replay angle inside the material layer of the probe beam and $d$ is the thickness of the material layer. The diffraction efficiency of an unslanted transmission holographic grating is found using

$$
\eta(t)=\frac{I_{\mathrm{d}}(t)}{I_{\text {in }} \times \text { F.C. }},
$$

where $I_{\mathrm{d}}$ is the probe diffracted beam intensity, $I_{\text {in }}$ is the input probe beam intensity and F.C. corrects for the Fresnel boundary reflections [18].

3.1.1. Reflection holography. In order to test the effects of chain transfer agent at high spatial frequencies, holographic gratings were also recorded in the reflection geometry. The spatial frequency of a holographic grating is determined by the wavelength and incidence angle of the recording beams. The spatial frequency for an unslanted reflection geometry grating is given by [19]

$$
f=\frac{2 n \cos \theta_{\mathrm{B}}}{\lambda},
$$

where $n$ is the average refractive index of the photopolymer layer, i.e., $n_{\text {dark }}, \lambda$ is the wavelength of the recording beams and $\theta_{\mathrm{B}}$ is the Bragg angle. Two spatial frequencies were recorded, $f=4286$ and 4799 lines $\mathrm{mm}^{-1}$. In order to simplify the measurement of the diffractive light in the reflection geometry, a slightly slanted grating of slant $\theta_{\mathrm{s}} \approx 5^{\circ}$ was used which sufficiently separated the diffracted and specularly reflected beams [18]. It is useful to examine this case in order to explore the effectiveness of the addition of chain transfer agents in situations involving very high spatial frequency exposures.

We note that the calculation of refractive index modulation for slanted reflection holographic gratings from diffraction efficiency measurements is different from that for unslanted 
Table 4. Experimental results of diffraction efficiency and thickness at different spatial frequencies for standard material, standard material with sodium formate (CTA-1) and standard material with 1-mercapto-2-proponal (CTA-2).

\begin{tabular}{llllllll}
\hline \multirow{2}{*}{$\begin{array}{l}\text { Spatial frequency } \\
\left(\text { lines } \mathrm{mm}^{-1} \text { ) }\right.\end{array}$} & \multicolumn{3}{c}{ Diffraction efficiency $\eta(\%)$} & & \multicolumn{3}{c}{ Thickness $d(\mu \mathrm{m})$} \\
\cline { 2 - 3 } \cline { 7 - 8 } & Standard & CTA-1 & CTA-2 & & Standard & CTA-1 & CTA-2 \\
\hline 500 & 89.16 & 89.57 & 91.33 & & 120 & 115 & 125 \\
1000 & 89.05 & 90.09 & 86.98 & & 118 & 112 & 115 \\
1428 & 91.56 & 90.35 & 90.51 & & 104 & 106 & 112 \\
2000 & 88.15 & 86.12 & 90.35 & 109 & 112 & 107 \\
2500 & 73.54 & 79.58 & 84.57 & 107 & 102 & 99 \\
3000 & 70.33 & 79.58 & 77.49 & 107 & 95 & 97 \\
4286 & 12.60 & 18.07 & 16.00 & 106 & 109 & 100 \\
4799 & 3.00 & 4.00 & 4.20 & 112 & 106 & 103 \\
\hline
\end{tabular}

transmission recording, equation (8). However, for the sake of simplicity, we treat the experimental results obtained in this case as unslanted, since the slant angle used is very small. From Kogelnik's coupled wave theory [17] the diffraction efficiency $\eta(t)$ for unslanted reflection geometry grating is given by

$$
\eta=t h^{2}\left(\frac{\pi n_{1} d}{\lambda \cos \theta_{\mathrm{B}}}\right),
$$

where $\lambda_{\mathrm{p}}$ is the replay probe wavelength, $\theta_{\mathrm{B}}$ is the on-Bragg replay angle inside the material layer and $d$ is the thickness of the material layer.

\subsubsection{Angular scan. Angular scans were carried out after} grating growth was complete, i.e., once the diffracted intensity reached its saturation value. The results are sinc squared type curves, as predicted by Kogelnik [17]. Using a fitting algorithm to fit Kogelnik's' analytic expression to the angular scan data, an estimate of the approximate material layer thickness and the saturation modulation are extracted [17, 20]. During fabrication, every attempt is made to ensure that the thicknesses of the layers used are all similar. We present the experimental results and fits for the standard material at four particular spatial frequencies in figure 2 and note that the thicknesses of layers estimated using this technique are all consistently similar, see table 4 . It can be observed that the width of the first-order diffraction becomes narrower as the grating spatial frequency increases. Changes in the grating thickness can occur due to swelling or shrinkage during and post exposure layer $[18,21]$. However, in this paper, we assume that the thickness remains constant throughout the course of examination, i.e., shrinkage and swelling effects are negligible, and is given by the thickness values extracted from the fits to angular scans, as presented in table 4 . Combining these results using equation (8), the temporal evolution of $n_{1}$ can be estimated. As can be seen in table 4, the diffraction efficiency achieved for each material composition under examination falls off for higher spatial frequencies. However, the falloff in diffraction efficiency for the material layers containing CTA is much less than in the standard material. Therefore an improvement of refractive index modulation at high spatial frequencies occurs in the photopolymer material containing CTAs.

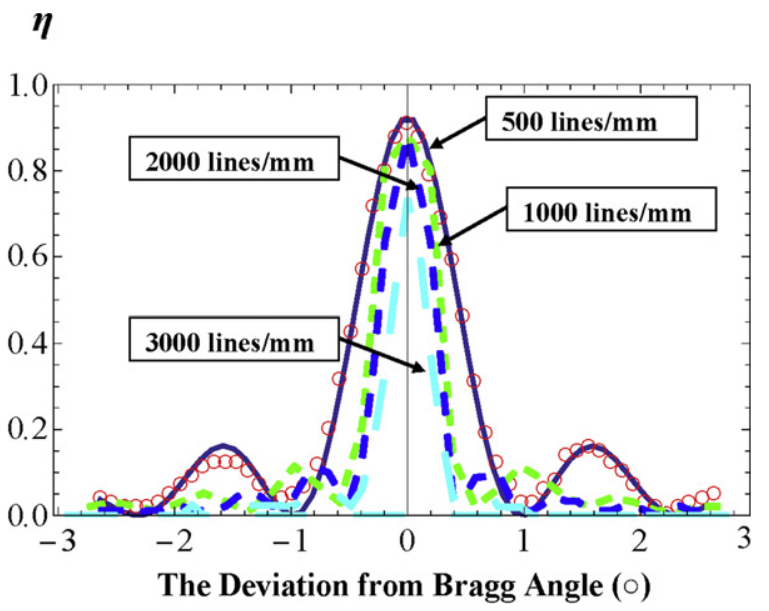

Figure 2. Plotted angular scan results of standard material for different spatial frequencies, for 500 (empty circles), 1000 (short dashed line), 2000 (long dashed line) and 3000 lines $\mathrm{mm}^{-1}$ (very long dashed line) and fit for 500 lines $\mathrm{mm}^{-1}$ (jointed line).

\subsection{Experimental results and analysis}

In the same manner as described in [22, 24], the absorption parameters were obtained from the fits to the normalized transmitted recording beams captured during the recording of the holographic gratings. These parameters determine the absorption behaviour of the materials and therefore the generation of the initiator radicals. The values estimated are $\varepsilon=1.43 \times 10^{8} \mathrm{~cm}^{2} \mathrm{~mol}^{-1}, \phi=0.018$, and $T_{\text {sf }}=0.76$, and are all in agreement with previously estimated values in the literature [2, 10, 22-24]. The exposing fringe visibility was $V=1$ and the relationships assumed between the rate constants used were $k_{\mathrm{p}}=k_{\mathrm{i}}$ and $k_{\mathrm{tp}}=k_{\mathrm{t}} \times 10$ [2, 9, 10,22-24].

In order to accurately represent the growth curves of refractive index modulation obtained for each spatial frequency and each material composition, average growth curves with appropriate error bars were generated from all the repeated experimental data sets and were used during the fitting procedure. Applying the extended NPDD model developed in part I [1], the experimental growth curve data were then fitted using a least squares algorithm, in which the mean square error (MSE) between the prediction of the model and the experimental data was minimized to extract key material parameters. In order to carry out the fitting process, search 
Table 5. Spatial frequency parameter estimations for standard AA/PVA material.

\begin{tabular}{|c|c|c|c|c|c|}
\hline $\begin{array}{l}\mathrm{SF}\left(\text { lines } \mathrm{mm}^{-1}\right) \\
{\left[n_{1}^{\text {sat }} \times 10^{-3}\right]}\end{array}$ & $k_{\mathrm{p}}\left(\times 10^{7}\right)$ & $k_{\mathrm{t}}\left(\times 10^{8}\right)$ & $D_{\mathrm{m} 0}\left(\times 10^{-10}\right)$ & $\sqrt{\sigma}(\mathrm{nm})$ & $\operatorname{MSE}\left(\times 10^{-10}\right)$ \\
\hline $500[2.07]$ & 2.7 & 3.0 & 3.0 & 60 & 1.96 \\
\hline $1000[2.20]$ & 2.3 & 3.6 & 1.0 & 68 & 1.64 \\
\hline $1428[2.36]$ & 2.8 & 3.0 & 3.0 & 55 & 0.89 \\
\hline 2000 [1.97] & 2.2 & 3.8 & 2.0 & 60 & 1.09 \\
\hline $2500[1.56]$ & 2.7 & 3.1 & 3.0 & 65 & 1.21 \\
\hline 3000 [1.38] & 2.6 & 3.2 & 3.0 & 60 & 2.72 \\
\hline Mean & $2.6 \pm 0.4$ & $3.3 \pm 0.5$ & $2.5 \pm 1.5$ & $61.3 \pm 6.7$ & $1.59 \pm 1.13$ \\
\hline
\end{tabular}

Table 6. Parameter estimations for spatial frequencies in AA/PVA with sodium formate (CTA-1) material.

\begin{tabular}{llllllll}
\hline $\begin{array}{l}\mathrm{SF}(\text { lines mm } \\
{\left[n_{1}^{\text {sat }} \times 10^{-3}\right]}\end{array}$ & $k_{\mathrm{p}}\left(\times 10^{7}\right)$ & $k_{\mathrm{t}}\left(\times 10^{8}\right)$ & $D_{\mathrm{m} 0}\left(\times 10^{-10}\right)$ & $k_{\mathrm{tr}}\left(\times 10^{6}\right)$ & $k_{\mathrm{ri}}\left(\times 10^{7}\right)$ & $\sqrt{\sigma}(\mathrm{nm})$ & $\mathrm{MSE}\left(\times 10^{-10}\right)$ \\
\hline $500[1.90]$ & 3.7 & 3.3 & 5.0 & 5.0 & 2.6 & 50 & 1.64 \\
$1000[2.18]$ & 3.5 & 3.4 & 3.0 & 2.0 & 1.0 & 51 & 2.72 \\
$1428[2.39]$ & 3.8 & 3.0 & 6.0 & 3.0 & 1.3 & 45 & 2.65 \\
$2000[2.12]$ & 3.2 & 4.0 & 6.0 & 2.0 & 1.7 & 45 & 2.36 \\
$2500[1.87]$ & 3.7 & 3.0 & 3.0 & 3.0 & 1.0 & 49 & 3.92 \\
$3000[1.60]$ & 3.8 & 3.6 & 1.0 & 2.0 & 1.0 & 47 & 1.17 \\
Mean & $3.6 \pm 0.4$ & $3.4 \pm 0.6$ & $4.0 \pm 3.0$ & $2.8 \pm 2.2$ & $1.4 \pm 1.2$ & $47.6 \pm 3.4$ & $2.41 \pm 1.24$ \\
\hline
\end{tabular}

Table 7. Parameter estimations for spatial frequencies in AA/PVA with 1-mercapto-2-propanol (CTA-2) material.

\begin{tabular}{llllllll}
\hline $\begin{array}{l}\mathrm{SF}(\text { lines mm } \\
{\left[n_{1}^{\text {sat }} \times 10^{-3}\right]}\end{array}$ & $k_{\mathrm{p}}\left(\times 10^{7}\right)$ & $k_{\mathrm{t}}\left(\times 10^{8}\right)$ & $D_{\mathrm{m} 0}\left(\times 10^{-10}\right)$ & $k_{\mathrm{tr}}\left(\times 10^{7}\right)$ & $k_{\mathrm{ri}}\left(\times 10^{6}\right)$ & $\sqrt{\sigma}(\mathrm{nm})$ & $\operatorname{MSE}\left(\times 10^{-10}\right)$ \\
\hline $500[1.97]$ & 2.0 & 3.5 & 5.0 & 1.0 & 8.0 & 40 & 1.98 \\
$1000[2.09]$ & 1.7 & 3.8 & 3.0 & 2.0 & 6.0 & 40 & 1.71 \\
$1428[2.38]$ & 1.6 & 3.9 & 2.0 & 2.0 & 3.0 & 42 & 1.53 \\
$2000[2.23]$ & 1.7 & 3.7 & 6.0 & 3.0 & 2.0 & 40 & 1.22 \\
$2500[2.03]$ & 2.1 & 3.0 & 2.0 & 1.0 & 3.0 & 38 & 5.53 \\
$3000[1.68]$ & 1.7 & 3.8 & 2.0 & 1.0 & 8.0 & 39 & 1.12 \\
Mean & $1.8 \pm 0.3$ & $3.6 \pm 0.6$ & $3.3 \pm 2.7$ & $1.7 \pm 1.3$ & $5.0 \pm 3.0$ & $39.8 \pm 2.2$ & $2.18 \pm 3.35$ \\
\hline
\end{tabular}

ranges of typical parameter values based on data presented in the literature were used $[2,7,14,16,22-26]$. The model was then applied to analyse the $n_{1}$ temporal growth curves for each material at each spatial frequency. In this way the monomer diffusion constant, $D_{\mathrm{m} 0}$, the propagation rate constant, $k_{\mathrm{p}}$, the initial rate, $k_{\mathrm{i}}$, the bimolecular termination rate constant, $k_{\mathrm{t}}$, the primary termination rate, $k_{\mathrm{tp}}$, the chain transfer rate, $k_{\mathrm{tr}}$, the reinitiation rate, $k_{\mathrm{ri}}$, and the non-local response length, $\sqrt{\sigma}$, were all estimated.

The parameters estimated for the compositions studied are presented in table 5 (standard), table 6 (CTA-1) and table 7 (CTA-2). For each spatial frequency the saturation refractive index modulation value, $n_{1}^{\text {sat }}$, is provided in the first column of each table.

Examining the estimated values for $k_{\mathrm{p}}, k_{\mathrm{t}}$ and $D_{\mathrm{m} 0}$ given in the three tables, it can be seen that they are consistent and close to the values previously reported in the literature $[2,7,16,22-25]$. In particular we note that the mean values estimated for $D_{\mathrm{m} 0}$ given in all the tables are very similar [27, 28].

The values obtained for the parameters $k_{\text {tr }}$ and $k_{\mathrm{ri}}$ in tables 6 and 7 lie in reasonable ranges based on the values reported in the literature $[14,26]$. It should be noted that the present model does not take account of the time varying viscosity effects occurring in the material due to polymerization of the monomer and all kinetic rates are assumed constant. Therefore, the kinetic parameters extracted from fits to the experimental data are in fact average values obtained over the course of grating growth. It is known that the kinetic rate constants can in some cases vary by orders of magnitude throughout the conversion of monomer to polymer [9]. These effects can explain the deviation observed in the mean values presented in table 7 . However, the values of kinetic parameters extracted by fitting the data vary within physically reasonable ranges and clearly indicate the different physical properties of the two CTAs when used in an AA/PVA based photopolymer material. For each CTA examined it should also be noted that the values found for $k_{\text {tr }}$ and $k_{\text {ri }}$ are consistent for each of the different spatial frequencies.

In table 5 the mean non-local response length in the standard AA/PVA material is estimated to be approximately $\sqrt{\sigma} \approx 61.3 \mathrm{~nm}$. This value agrees well with the previous estimates reported in the literature $[2,25]$. For the material containing sodium formate (CTA-1) the corresponding value is $\sqrt{\sigma} \approx 47.6 \mathrm{~nm}$, as given in table 6 , which corresponds to a reduction $\sim 22.3 \%$ in the mean $\sqrt{\sigma}$ value. In table 7 , the $\sqrt{\sigma}$ value obtained for the material with 1-mercapto-2-propanol (CTA-2) is $\sqrt{\sigma} \approx 39.8 \mathrm{~nm}$. This corresponds to an $\sim 35.0 \%$ 


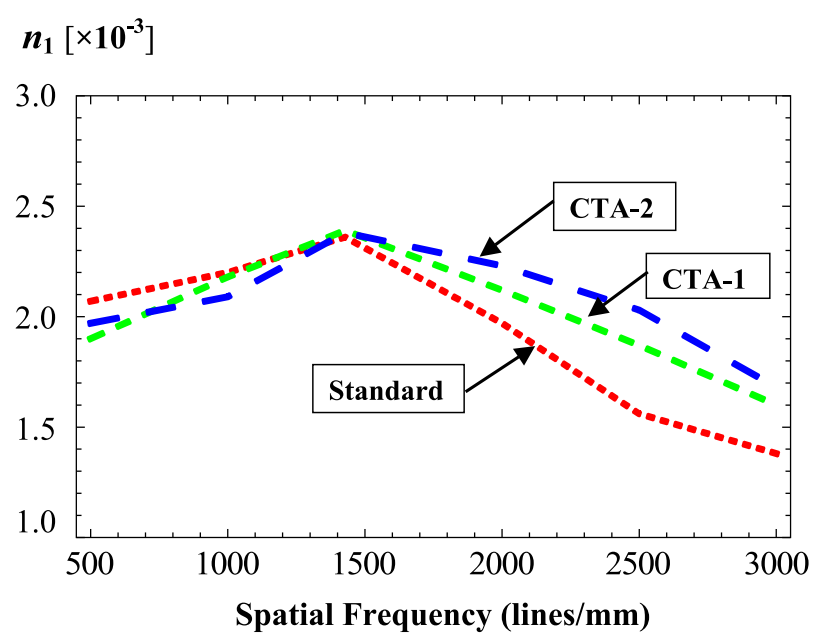

Figure 3. Experimental results for the spatial frequency response of the saturation refractive index modulation, $n_{1}$, for standard material (short dashed line), standard material with $0.96 \mathrm{~g}$ sodium formate (CTA-1) (medium dashed line) and standard material with $0.53 \mathrm{ml}$ 1-mercapto-2-propanol (CTA-2) (long dashed line).

reduction in the mean $\sqrt{\sigma}$ value. Therefore significant improvements in the high spatial frequency responses with the addition of CTA can be clearly observed. CTA-2 is more effective in reducing the average polymer chain length, which is also consistent with the results presented in section 2 . For the reflection geometry holographic recording (very high spatial frequency), by processing the results in table 4 using equation (11), the values of $n_{1}$ for standard, CTA-1, CTA-2 can be estimated as follows: (i) in the case of 4286 lines $\mathrm{mm}^{-1}$, $n_{1}=\{0.58,0.67,0.69\} \times 10^{-3}$; and (ii) in the case of 4799 lines $\mathrm{mm}^{-1}, n_{1}=\{0.27,0.33,0.35\} \times 10^{-3}$. These results consistently indicate an accompanying improvement in the magnitude of the saturation refractive index modulation. Based on the analysis above and the demonstration in [2] it is clear that it is the shortening of the polymer chains which most consistently explains the observed improvement in the spatial frequency responses.

In order to more clearly examine the spatial frequency response for each material under examination, figure 3 shows the results listed in tables 5, 6 and 7. The improvements in the high spatial frequency response can clearly be observed. In order to demonstrate the improvements in AA/PVA material performance, numerical fits were carried out to the growth curves of all the three tested samples, for the 3000 lines $\mathrm{mm}^{-1}$ spatial frequency case. The results are presented in figure 4 with the associated error bars, indicating the reproducibility of the experimental results.

\section{Conclusion}

In this paper, part II, an extended NPDD model, which includes the effect of a chain transfer agent in the kinetics of freeradical photo-polymerization [1], and also includes most of the other major photochemical reactions and mass transport effects occurring during the holographic grating formation, has

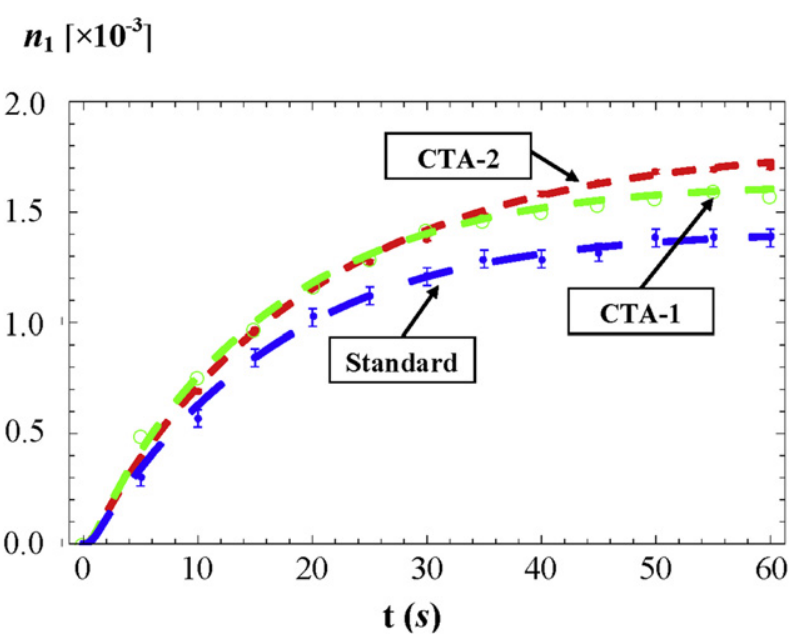

Figure 4. Experimental growth curve data with error bars and fits at 3000 lines $\mathrm{mm}^{-1}$, for standard material (long dashed line), with sodium formate (CTA-1) (medium dashed line and empty circle points) and with 1-mercapto-2-propanol (CTA-2) (short dashed line).

been experimentally examined. The prediction of this model, that a decrease in the non-local response length will cause an improvement in the high spatial frequency response of the photopolymer material, was explored. A number of material layers made using various concentrations of two CTAs, i.e., sodium formate and 1-mercapto-2-propanol, were examined by recording growth curves at a range of spatial frequencies. These curves were then numerically fitted with the proposed NPDD model and key parameters were extracted to quantify the effects on the non-local response length and hence the material's spatial frequency response.

To examine the effect of the addition of transfer agent on the average polymer chain length, a diffusion based technique was developed and the results presented. It was assumed for simplicity that the polymer chains formed during recording could be grouped into two subsets having different average molecular weights in order to characterize polymer chain diffusion in a non-cross-linked photopolymer system. In this way, a comparison of polymer diffusion rates in standard material and material containing different types and different concentrations of chain transfer agents was obtained. Thus, the most appropriate volume fraction for each transfer agent to be used was explored.

In order to experimentally validate the prediction of the model in section 2, it was applied to fit experimental data in three types of material and material parameters were estimated. The improvement in spatial frequency response of an AA/PVA photopolymer through the addition of a chain transfer agent has been demonstrated. This improvement is due to the effect of transfer agent in decreasing the average length of the polymer chains formed, which contributes to the increased localization of the polymerization. The most effective material combination used resulted in a reduction in the non-local parameter from 61 to $41 \mathrm{~nm}$. Comparing the reduction of $\sqrt{\sigma}$ achieved by the two types of transfer agents, 1mercapto-2-propanol has been shown to be better than sodium 
formate. Furthermore, other key material kinetics parameters were extracted by fitting the experimental data, including the rate constants of chain transfer and re-initiation; thus the kinetic behaviour of transfer agents has been quantified. The predictions and results in this series of papers indicate that a particular type of CTA with a higher rate constant of chain transfer will produce a lower average polymer chain length and therefore a reduction in the non-local response length. The low MSE values achieved and good general agreement between parameters extracted using different experimental data sets indicate the consistent quality of the predictions of the model. Future work will include the derivation of a more accurate formula expressing the relationship between the number average degree of polymerization, $D P_{n}$, and the nonlocal response length, $\sqrt{\sigma}$. Also, inclusion of the time varying viscosity effects of the material during monomer conversion is necessary in order to improve the NPDD model's predictive behaviour [29].

\section{Acknowledgments}

One of the authors (JG) currently holds an IRCSET Government of Ireland Postgraduate Scholarship in Science, Engineering and Technology, while another author (MRG) currently holds a Government of Ireland Post-doctoral Scholarship in Science, Engineering and Technology, both granted by the Irish Research Council for Science, Engineering and Technology. We would also like to acknowledge the support of Enterprise Ireland and Science Foundation Ireland under the National Development Fund.

\section{References}

[1] Guo J, Gleeson M R, Liu S and Sheridan J T 2011 J. Opt. 13095601

[2] Gleeson M R, Sabol D, Liu S, Close C E, Kelly J V and Sheridan J T 2008 Improvement of the spatial frequency response of photopolymer materials by modifying polymer chain length J. Opt. Soc. Am. B 25 396-406

[3] Fevola M, Hester R and McCormack C 2003 Molecular weight control of polyacyrlamide with sodium formate as a chain-transfer agent: characterization via size exclusion chromatography/multi-angle laser light scattering and determination of chain-transfer constant J. Polym. Sci. A 41 560-8

[4] Valdebenito A and Encinas M V 2005 Thiophenols as chain transfer agents in the polymerization of vinyl monomers Polymer 46 10658-62

[5] Karasu F, Arsu N and Yagci Y 2007 2-mercapto thioxanthone as a chain transfer agent in free-radical polymerization J. Appl. Polym. Sci. 103 3766-70

[6] www.arkema-inc.com/index.cfm

[7] Lawrence J R, O’Neill F T and Sheridan J T 2001 Photopolymer holographic recording material Optik 112 449-63

[8] Kelly J V, Gleeson M R, Close C E, O’Neill F T, Sheridan J T, Gallego S and Neipp C 2005 Temporal analysis of grating formation in photopolymer using the nonlocal polymerization-driven diffusion model Opt. Express 13 6990-7004

[9] Gleeson M R and Sheridan J T 2009 Non-local photo-polymerization kinetics including multiple termination mechanisms and dark reactions: part I. Modelling J. Opt. Soc. Am. B 26 1736-45

[10] Gleeson M R, Liu S, McLeod R R and Sheridan J T 2009 Non-local photo-polymerization kinetics including multiple termination mechanisms and dark reactions: part II. Experimental validation J. Opt. Soc. Am. B 26 1746-54

[11] Aubrecht I, Miler M and Koudela I 1998 Recording of holographic diffraction gratings in photopolymers: theoretical modelling and real-time monitoring of grating growth J. Mod. Opt. 45 1465-77

[12] Mayo F R 1943 Chain transfer in the polymerization of styrene: the reaction of solvents with free radicals $J$. Am. Chem. Soc. 65 2324-9

[13] Gartner H A 1992 US Patent Specification 5,171,783

[14] Odian G 1991 Principles of Polymerization 4th edn (New York: Wiley)

[15] Gleeson M R, Kelly J V, O’Neill F T and Sheridan J T 2005 Recording beam modulation during grating formation Appl. Opt. 44 5475-82

[16] Gleeson M R, Kelly J V and Sheridan J T 2007 Modelling the photochemical effects present during holographic grating formation in photopolymer materials J. Appl. Phys. 102 1-9

[17] Kogelnik H 1969 Coupled wave theory for thick holographic gratings Bell Syst. Tech. J. 48 2909-47

[18] O’Neill F T, Lawrence J R and Sheridan J T 2001 Thickness variation of a self-processing acrylamide-based photopolymer and reflection holography Opt. Eng. 40 533-9

[19] Kim J M, Choi B S, Choi Y S, Kim J M, Bjelkhagen H I and Phillips N J 2002 Holographic optical elements recorded in silver halide sensitized gelatin emulsions: part 2. Reflection holographic optical elements Appl. Opt. 41 1522-33

[20] Neipp C, Belendez A, Gallego S, Ortuno M, Pascual I and Sheridan J 2003 Angular responses of the first and second diffracted orders in transmission diffraction grating recorded on photopolymer material Opt. Express 11 1835-43

[21] O'Neill F T, Carr A J, Daniels S M, Gleeson M R, Kelly J V, Lawrence J R and Sheridan J T 2005 Refractive elements produced in photopolymer layers J. Mater. Sci. 40 4129-32

[22] Gleeson M R, Liu S, Guo J and Sheridan J T 2010 Non-Local photo-polymerization kinetics including multiple termination mechanisms and dark reactions: part III. Primary radical generation and inhibition J. Opt. Soc. Am. B 27 1804-12

[23] Liu S, Gleeson M R, Guo J and Sheridan J T 2010 Optical characterization of photopolymers materials: theoretical and experimental examination of primary radical generation Appl. Phys. B 100 559-69

[24] Liu S, Gleeson M R, Guo J and Sheridan J T 2010 High intensity response of photopolymer materials for holographic grating formation Macromolecules 43 9462-72

[25] Wu S and Glytsis E N 2003 Holographic grating formation in photopolymers: analysis and experimental results based on a nonlocal diffusion model and rigorous coupled-wave analysis J. Opt. Soc. Am. B 20 1177-88

[26] Brandrup J, Immergut E H and Grulke E A 1999 Polymer Handbook 4th edn (New York: Wiley)

[27] Close C E, Gleeson M R and Sheridan J T 2011 Monomer diffusion rates in photopolymer material. Part I. Low spatial frequency holographic gratings $J$. Opt. Soc. Am. B 28 658-66

[28] Close C E, Gleeson M R, Mooney D A and Sheridan J T 2011 Monomer diffusion rates in photopolymer material. Part II. High-frequency gratings and bulk diffusion J. Opt. Soc. Am. B $28842-50$

[29] Gleeson M R, Guo J and Sheridan J T 2011 Optimisation of photopolymers for holographic applications using the non-local photo-polymerization driven diffusion model $O p t$. Express at press 